\title{
Deletion of Protein Tyrosine Phosphatase Nonreceptor Type 2 in Intestinal Epithelial Cells Results in Upregulation of the Related Phosphatase Protein Tyrosine Phosphatase Nonreceptor Type 23
}

\author{
Sima Ulugöla Larissa Hering ${ }^{a}$ Roberto Manzini ${ }^{a} \quad$ Ana Montalban Arques ${ }^{a}$ \\ Claudia Gottier $^{a}$ Silvia Lang ${ }^{a} \quad$ Michael Scharl ${ }^{a}$ b Marianne R. Spalinger ${ }^{a}$ \\ ${ }^{a}$ Department of Gastroenterology and Hepatology, University Hospital Zurich, University of Zurich, Zurich, \\ Switzerland; ' Zurich Institute for Human Physiology, University of Zurich, Zurich, Switzerland
}

\section{Keywords}

Tyrosine phosphatases · HD-PTP · T cell protein tyrosine phosphatase $\cdot$ Intestinal inflammation $\cdot$ Intestinal epithelial cells

\begin{abstract}
Background/Aims: Knockdown of protein tyrosine phosphatase nonreceptor type 2 (PTPN2) exaggerates IFN- $\gamma^{-}$ induced intestinal barrier defects, but mice constitutively lacking PTPN2 in epithelial cells (PTPN2xVilCre mice) do not show changes in epithelial function or enhanced susceptibility to experimental colitis. Here, we investigated whether PTPN2 modulates the expression of related tyrosine phosphatases. Methods: PTPN2 knockdown in HT-29 cells was induced using siRNA constructs. Acute colitis in PTPN2xVilCre mice was induced by $2 \%$ dextran sulfate sodium (DSS) in drinking water for 7 days. Colitis-associated tumors were induced by injection of azoxymethane prior to treatment with DSS for 3 consecutive cycles. Results: In HT-29 cells, PTPN2 depletion resulted in enhanced mRNA expression of PTPN11 and PTPN23 and in parallel to upregulation of IL-18 mRNA upon treatment with TNF for $24 \mathrm{~h}$. DSS treatment of PTPN2deficient mice resulted in a strong induction of Ptpn 23 mRNA
\end{abstract}

\section{KARGER}

(c) 2019 S. Karger AG, Basel

E-Mail karger@karger.com

www.karger.com/iid in colon tissue in vivo. In the tumor model, Ptpn23 mRNA was again clearly upregulated in nontumor tissue from PTPN2deficient mice; however, this was not observed in tumor tissue. Conclusions: Our experiments show that PTPN23 function might, at least partially, compensate lack of PTPN2 in epithelial cells. Upregulation of PTPN23 might therefore crucially contribute to the lack of a colitis phenotype in PTPN2VilCre mice.

C 2019 S. Karger AG, Basel

\section{Introduction}

Inflammatory bowel disease (IBD) is characterized by a chronic, relapsing, and remitting inflammation of the gastrointestinal tract. Patients suffer from abdominal pain, diarrhea, and extraintestinal manifestations, including skin rashes, uveitis, and arthritis, are common complications. Further, patients with longstanding disease course might be at a higher risk of developing both, gastrointestinal as well as extraintestinal malignancies, including skin cancer, lymphoma, and colorectal cancer [1]. The increased risk to develop colorectal cancer is primarily due to the fact that chronic inflammation and re- 
current epithelial defects might promote aberrant proliferation and malignant transformation of intestinal epithelial cells (IECs) [2].

Although the exact etiopathogenesis of IBD has not been completely understood, it is commonly accepted that genetic, immunological, environmental, and bacterial factors play a key role in the development of chronic intestinal inflammation [3]. Genome-wide association studies identified 240 genetic loci that affect the susceptibility to develop IBD, including protein tyrosine phosphatase nonreceptor type 2 (PTPN2, also referred to as T cell protein tyrosine phosphatase [PTP]) [4-6]. Variants in the gene locus encoding PTPN2 are associated with both, CD and UC [7]. PTPN2 is a member of the PTPs family, which, together with protein tyrosine kinases, are responsible for regulating the phosphorylation status of intracellular proteins [8]. Phosphorylation and dephosphorylation of protein tyrosine residues are essential mechanisms for activating or deactivating intracellular signaling cascades.

PTPN2 is expressed in all tissues of the body, and in humans 2 splicing variants with slightly different subcellular localization patterns have been documented [9]. Since PTPN2 exerts obviously important anti-inflammatory functions, genetic deletion of PTPN2 in mice leads to death not later than 5 weeks after birth as a result of systemic inflammation with signs of severe colitis [10].

IECs form a tight intestinal epithelial barrier to prevent food particles and intestinal bacteria from unlimited access to the host [11]. In IBD patients, defects in the epithelial barrier and increased intestinal permeability have been well documented $[12,13]$. An important cytokine that modulates intestinal permeability is IFN- $\gamma$ [14]. Of note, IFN- $\gamma$ also stimulates PTPN2 mRNA and protein expression, and PTPN2 in turn negatively regulates IFN$\gamma$-induced signaling cascades and dampens IFN- $\gamma$ induced barrier defects [15]. Elevated PTPN2 mRNA and protein levels are found in colonic tissue samples of CD patients with acute inflammation, but not in samples of CD patients in remission [16]. PTPN2 knockdown in cell culture experiments exaggerates IFN- $\gamma$-induced epithelial barrier permeability and promotes pro-inflammatory cytokine secretion [15]. These in vitro findings suggest that PTPN2 is important for the preservation of intestinal barrier functions, especially upon inflammatory insults. However, in vivo experiments from Kasper et al. [17] showed that mice that specifically lack PTPN2 in IECs do not show enhanced disease severity upon induction of acute or chronic dextran sulfate sodium (DSS)-mediated colitis. Of note, those mice exhibit increased IEC proliferation and faster intestinal wound healing.

These rather unexpected findings led us to the hypothesis that in mice lacking PTPN2 in the intestinal epithelium, up-regulated expression of other tyrosine phosphatases might compensate for the loss of PTPN2. Therefore, the aim of the presented work was to investigate whether loss of PTPN2 in IECs results in up-regulation of nonreceptor PTPs that dephosphorylate similar target molecules as PTPN2, which might compensate for PTPN2 deficiency.

\section{Material and Methods}

\section{Cell Culture and siRNA Transfections}

HT-29 cells were obtained from DSMZ (Braunschweig, Germany) and cultured as described previously [17] in culture medium (DMEM; Thermo Fisher Scientific, Waltham, MA, USA), supplemented with $1 \%$ nonessential amino acids (Thermo Fisher Scientific) and $10 \%$ FCS. For experiments, $1 \times 10^{6}$ cells were seeded in 12-well plates. For siRNA transfections, medium was changed to serum-free DMEM, and 50 pmol of 3 different PTPN2-specific siRNA constructs, or 50 pmol of 3 different nontargeting control siRNA constructs were mixed with RNAiMAX transfection reagent (Thermo Fisher Scientific) and applied to the cells. After $12 \mathrm{~h}$, the medium was changed for normal culture medium. Twenty-four hours after the transfection, cells were treated with $100 \mu \mathrm{g} /$ $\mathrm{mL}$ IFN- $\gamma$ or $100 \mu \mathrm{g} / \mathrm{mL}$ TNF, and RNA was harvested $24 \mathrm{~h}$ later.

\section{Mice}

C57/Bl6 mice with a floxed PTPN2 gene (PTPN2 fl/fl mice, termed wild-type throughout the manuscript) and PTPN2 fl/fl mice expressing Cre-recombinase under the Villin promoter (PTPN2xVilCre mice) that lack PTPN2 specifically in IECs [17] were used for all studies. Acute colitis was induced via administration of $2.5 \%$ DSS in the drinking water as described by Kasper et al. [17]. To induce colitis-associated tumors, mice were treated for 3 consecutive cycles consisting of 7 days with 1.5\% DSS followed by 10 days recovery phase each. At the first day of each cycle, mice were injected with $10 \mathrm{mg} / \mathrm{kg}$ body weight azoxymethane (AOM). All animal experiments were performed according to local animal welfare legislation and were approved by the Veterinary Office of the Canton Zurich (ZH-255/2014).

\section{RNA Isolation}

Tissue samples were frozen in liquid nitrogen and stored in $-80^{\circ} \mathrm{C}$ until RNA isolation. Cells were washed 2 times in ice cold PBS, lysed in $350 \mu \mathrm{L}$ RLT buffer, and homogenized using a $26 \mathrm{G}$ needle. Colon pieces were mechanically disrupted in $350 \mu \mathrm{L}$ RLT buffer and dithiothreitol (Qiagen, Düsseldorf, Germany) using the gentleMACS tissue disrupter according to the manufacturer's instructions. To isolate total RNA, the RNeasy Plus Mini Kit (Qiagen) was used according to the manufacturer's instructions. RNA concentration was measured with a spectrophotometer NanoDrop ND1000 (NanoDrop Technologies, Wilmington, NC, USA) by absorbance at $260 \mathrm{~nm}$. 
Reverse Transcription and Real-Time Polymerase Chain

Reaction

A total of $1 \mu \mathrm{g}$ RNA was transcribed to complementary DNA using the High-Capacity Complementary DNA Reverse Transcription Kit (Thermo Fisher Scientific, 4368813). Real-time polymerase chain reaction (PCR) was performed using FAST qPCR MasterMix for Taqman Assays (Thermo Fisher Scientific) on a Fast HT7900 Real-Time PCR system using SDS Software (Thermo Fisher Scientific) with the following amplification steps: Initial enzyme activation for $5 \mathrm{~min}$ at $95^{\circ} \mathrm{C}$, followed by 45 cycles of denaturing for $15 \mathrm{~s}$ at $95^{\circ} \mathrm{C}$ and annealing/extending for $1 \mathrm{~min}$ at $60^{\circ} \mathrm{C}$ each. Measurements were performed in triplicates using mouse $\beta$-actin as endogenous control. Results were analyzed using the $\Delta \Delta \mathrm{CT}$ method.

\section{Western Blotting}

For Western blot analyses, cells were washed 2 times in PBS prior to lysis in M-PER buffer (Thermo Fisher Scientific) according to the manufacturer's instructions. After $30 \mathrm{~min}$. lysates were centrifuged for $10 \mathrm{~min}$ at $17,000 \mathrm{~g}$ and protein-containing supernatants collected into fresh tubes. Aliquots containing equal amounts of protein were loaded on $10 \%$ polyacrylamide gels and proteins separated for $1 \mathrm{~h}$ prior to blotting onto nitrocellulose membranes. Membranes were blocked for $1 \mathrm{~h}$ at room temperature (RT) with $3 \%$ milk, $1 \%$ bovine serum albumin in Tris-buffered saline containing $1 \%$ Tween prior to incubation with anti-HDPTP (1:100, Atlas antibodies, Stockholm, Sweden), anti PTPN2 (1:500, Millipore-Merck, Burlington, MA, USA), anti-IL-18 (1:500; R\&D Systems, Minneapolis, MN, USA), or anti-b-actin (1:10,000; Millipore-Merck) antibodies overnight. After washing 3 times in Tris-buffered saline containing 1\% Tween, membranes were incubated with HRP-coupled secondary antibody (1:3,000; Santa Cruz Biotechnologies, Dallas, TX, USA) for $1.5 \mathrm{~h}$ and immunoreactive proteins detected using an ELC substrate (Witec AG, Sursee, Switzerland).

\section{Enzyme-Linked Immunosorbent Assay}

Antihuman and antimouse IL-18 Enzyme-Linked Immunosorbent Assay (ELISA) kits were purchased from R\&D systems, and $100 \mu \mathrm{L}$ cell culture supernatant or $50 \mu \mathrm{L}$ serum used for detection of IL-18 according to the manufacturer's instructions.

\section{Immunhistochemistry}

Immunhistochemistry (IHC) was performed on formalinfixed, paraffin-embedded tissue specimen using a peroxidasebased method with diaminobenzidine chromogen. Tissue samples were incubated with Histoclear (Chemie-Brunschwig, Basel, Switzerland) and descending concentrations of ethanol. Antigen retrieval was performed using citrate buffer, pH 6.0 (DAKO, Glostrup, Denmark) for $30 \mathrm{~min}$ at $98^{\circ} \mathrm{C}$. Endogenous peroxidases were deleted by incubation with $0.9 \%$ hydrogen peroxide for $15 \mathrm{~min}$ at $\mathrm{RT}$, and blocking was performed using $3 \%$ bovine serum albumin for $2 \mathrm{~h}$. Sections were stained overnight at $4{ }^{\circ} \mathrm{C}$ with an antimouse PTPN23 antibody (Novus biologicals, 1:50 dilution). HRP-labeled secondary antirabbit IgG-antibody (Vector laboratories, Peterborough, UK) was applied for $1 \mathrm{~h}$ at RT and antibody binding visualized by a liquid diaminobenzidine + Substrate Chromogen System (Vector laboratories). Then samples were counterstained with hematoxylin, dehydrated in ascending concentrated ethanol and histoclear solutions, and finally mounted. Microscopic assessment was done using an AxioCam HRc (Zeiss, Jena, Germany) on a Zeiss Axio Imager.Z2 microscope (Zeiss) with AxioVision Release 4.8.2 software (Zeiss).

\section{Statistics}

All statistical analyses were performed using GraphPad Prism 5 Software (GraphPad Software Inc., La Jolla, CA, USA). $p$ values below 0.05 were considered significant.

\section{Results}

\section{PTPN2 Controls the Expression of PTPN1, PTPN9} and PTPN23 in HT-29 Cells

We first investigated whether loss of PTPN2 in IECs might affect the expression of other tyrosine phosphatases with similar targets to PTPN2 or with reported functions in intestinal inflammation, namely, PTPN1 (also known as PTP1B), PTPN9, PTPN11, PTPN22, and PTPN23. To examine whether their mRNA expression is altered upon deletion of PTPN2 in an IEC line, we treated HT-29 colon carcinoma cells with PTPN2-specific smallinterfering (si) RNA or nontargeting control siRNA constructs. Cells were then treated with $100 \mu \mathrm{g} / \mathrm{mL}$ IFN- $\gamma$ or $100 \mu \mathrm{g} / \mathrm{mL}$ TNF for $24 \mathrm{~h}$. As expected, mRNA expression of PTPN2 was clearly reduced upon PTPN2-siRNA transfection showing that only $40-50 \%$ of the initial PTPN2 mRNA expression was still retained (Fig. 1a).

We found that TNF- $\alpha$, but not IFN- $\gamma$-treatment slightly enhanced the expression of PTPN1 mRNA (Fig. 1b), while loss of PTPN2 resulted in lower basal PTPN1 mRNA expression, and its induction was significantly decreased upon TNF- $\alpha$ treatment when compared to PTPN2-competent cells $(p<0.05)$. Of note, without treatment, PTPN9 mRNA expression was slightly but significantly enhanced upon knockdown of PTPN2 ( $p<0.01$, Fig. 1c). TNF treatment resulted in significantly reduced mRNA expression of PTPN9 in PTPN2 competent, as well as in PTPN2knockdown cells compared to their respective controls. PTPN2 knockdown resulted in higher PTPN9 mRNA expression already in untreated controls as well as in response to treatment with TNF- $\alpha$. PTPN11 mRNA expression was elevated in untreated PTPN2-knockdown cells when compared to untreated PTPN2-competent cells and TNF- $\alpha$ stimulation even further enhanced PTPN11 levels ( $p<0.001$, Fig. 1d), while IFN- $\gamma$ decreased PTPN1 1 mRNA levels in PTPN2-deficient cells. Further, IFN- $\gamma$ treatment promoted PTPN22 mRNA expression in PTPN2-deficient IECs ( $p<0.05$, Fig. 1e), whereas PTPN22 mRNA expression was not affected by TNF- $\alpha$ stimulation $(p<0.001)$.
Ulugöl et al. 


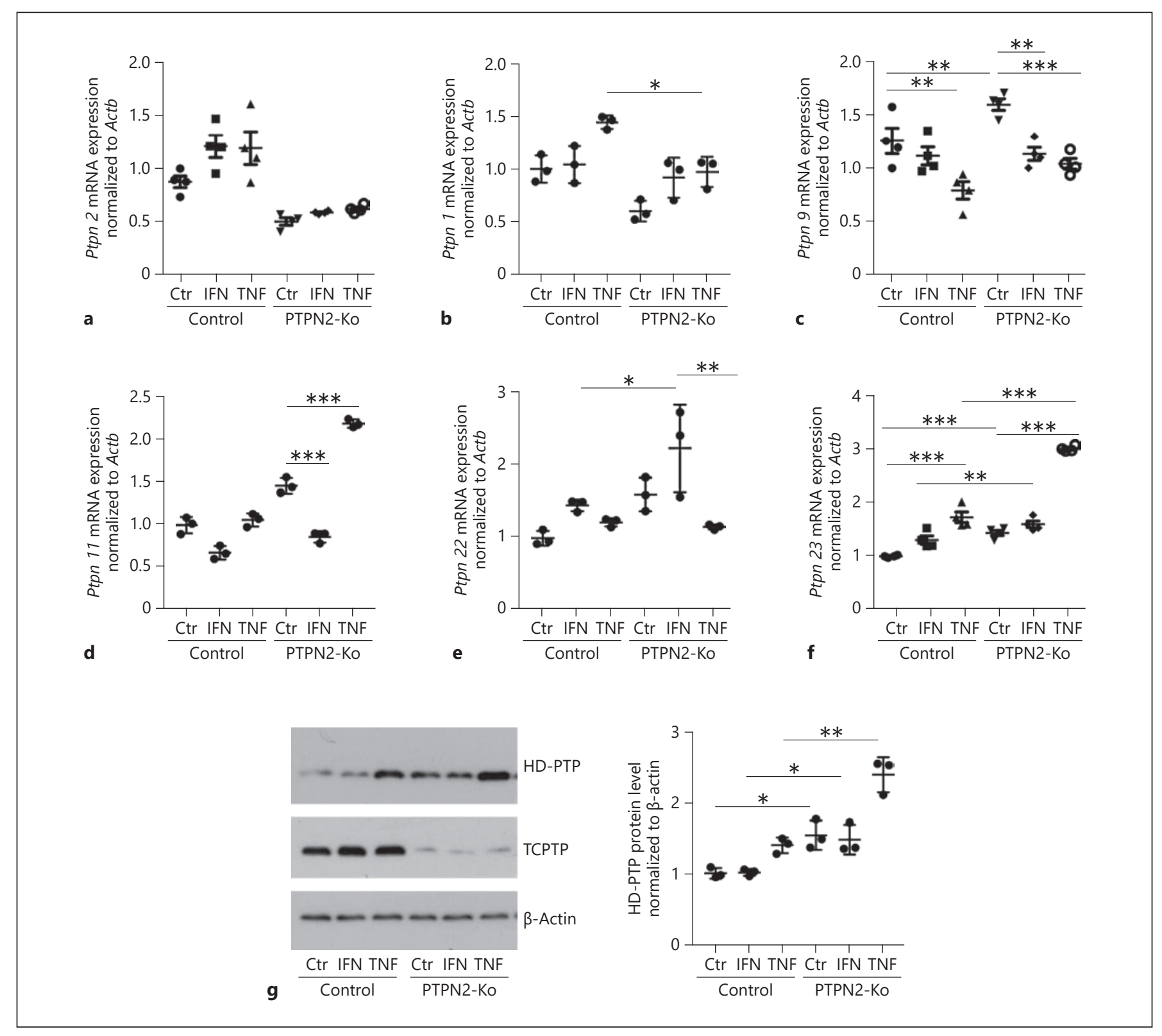

Fig. 1. Deletion of PTPN2 in HT-29 cells results in enhanced PTPN9, PTPN11, and PTPN23 expression. HT-29 cells were treated with nontargeting control siRNA or with PTPN2-specific siRNA and activated with $100 \mathrm{ng} / \mathrm{mL}$ IFN- $\gamma$ or $100 \mathrm{uL} / \mathrm{mL}$ TNF- $\alpha$ for $24 \mathrm{~h}$ as indicated. Cell lysates were analyzed for mRNA expression

Of note, basal levels of PTPN23 mRNA were significantly higher in PTPN2-knockdown cells when compared to PTPN2-competent HT-29 cells. This effect was clearly enhanced upon TNF- $\alpha$ treatment $(p<0.001$, Fig. 1f). TNF- $\alpha$ also induced PTPN23 mRNA levels in PTPN2-competent cells. Upregulation of HD-PTP (the protein product of $P T P N 23$ ) was confirmed by Western

Enhanced PTPN23 Expression Upon Deletion of PTPN2 of (a) PTPN2, (b) PTPN1, (c) PTPN9, (d) PTPN11, (e) PTPN22, and (f) PTPN23. Data were normalized to cells treated with control siRNA without activation. ${ }^{*}$ Significant differences $* p<0.05$, ${ }^{* *} p<0.01,{ }^{* * *} p<0.001$ Newman Keul's test; Ctr, Control; Actb, actin beta; PTPN, protein tyrosine phosphatase nonreceptor type.

blotting, where we also found clearly enhanced HDPTP levels upon treatment with TNF- $\alpha$ in PTPN2-competent cells, and increased basal levels of HD-PTP in PTPN2-deficient cells. Of note the TNF- $\alpha$-induced increase of HD-PTP was further potentiated in PTPN2deficient cells, confirming our findings on mRNA level (Fig. 1g). 


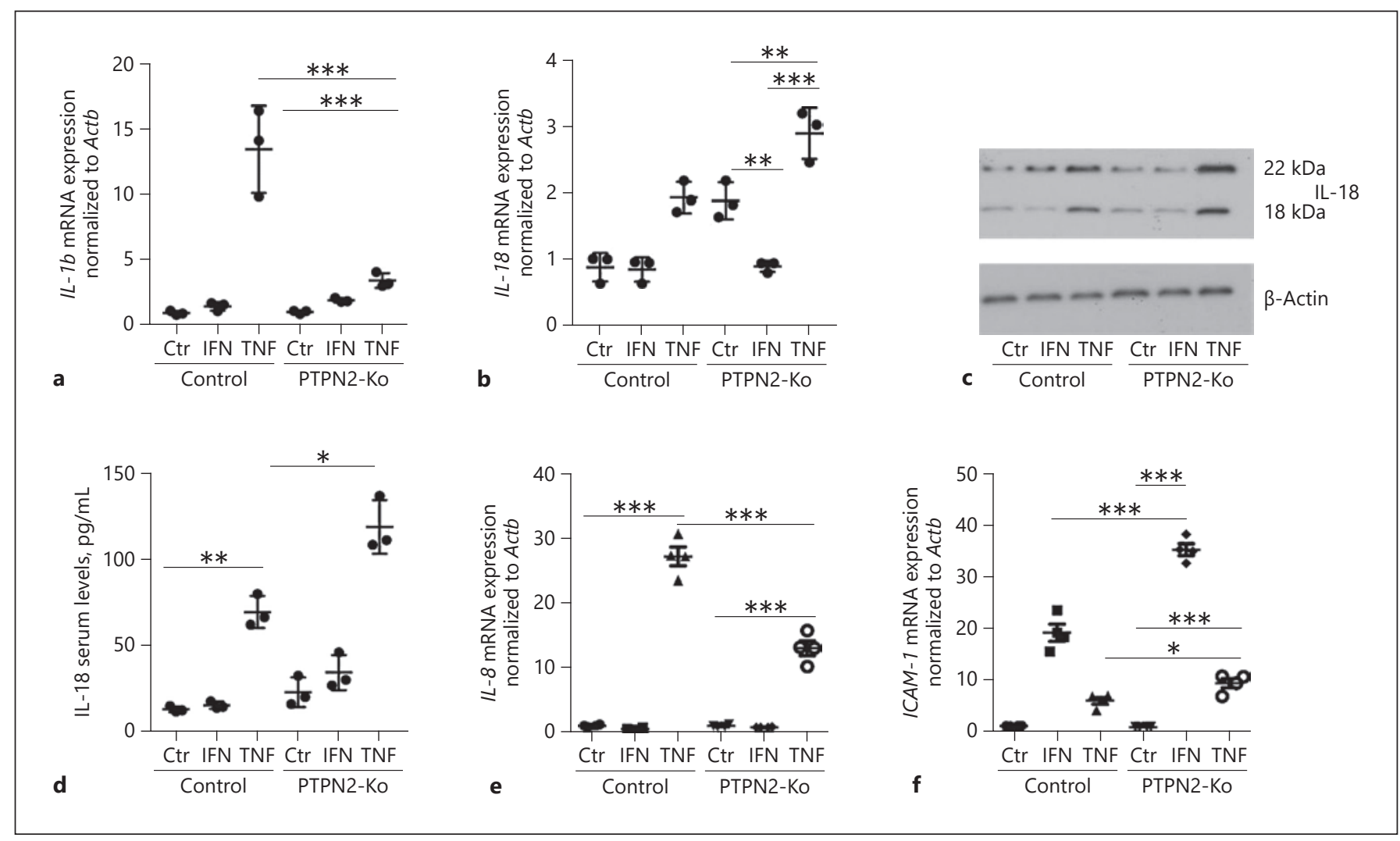

Fig. 2. Deletion of PTPN2 in HT-29 cells affects mRNA expression of proinflammatory cytokines. HT-29 cells were treated with nontargeting siRNA or with PTPN2-specific siRNA and activated with $100 \mathrm{ng} / \mathrm{mL}$ IFN- $\gamma$ or $100 \mathrm{uL} / \mathrm{mL}$ TNF- $\alpha$ for $24 \mathrm{~h}$ as indicated. Cell lysates were analyzed for mRNA expression of (a) IL1B, and (b) IL18. c, d Protein levels of IL-18 were analyzed by (c) Western blot and (d) ELISA. mRNA levels of (e) IL8, and (f) ICAM1 were analyzed by qPCR. Data were normalized to cells treated with control siRNA without activation. ${ }^{*}$ Significant differences $* p<0.05$, ${ }^{* *} p<0.01,{ }^{* * *} p<0.001$ Newman Keul's test; Ctr, Control; Actb, actin beta; PTPN, protein tyrosine phosphatase nonreceptor type.
PTPN2 Regulates the Expression of Pro-Inflammatory Cytokines in HT29 Cells

Having demonstrated that PTPN2 is involved in the regulation of the expression of mainly PTPN11 and PTPN23 in HT29 IEC, we next analyzed the mRNA expression of pro-inflammatory cytokines. TNF- $\alpha$ stimulated $I L-1 b$ expression in both, PTPN2-competent and PTPN2-siRNA treated HT29 cells, but this effect was clearly less pronounced in PTPN2-siRNA-transfected cells $(p<0.001$, Fig. 2a). IL-18 mRNA expression, on the otherhand, wasalso upregulated upon TNF- $\alpha$-stimulation in HT29 cells, and this effect was further enhanced in PTPN2-siRNA-treated cells $(p<0.001$, Fig. $2 b)$. Even without treatment, PTPN2-deficient cells showed highly increased $I L-18$ mRNA expression, which was clearly reduced in response to IFN- $\gamma$-stimulation $(p<0.01)$. Increased IL-18 levels were also confirmed in Western blot analyses (Fig. 2c) and IL-18 ELISA (Fig. 2d).
In addition, TNF- $\alpha$ stimulation resulted in highly increased mRNA expression of $I L-8 \mathrm{mRNA}$ in both, PTPN2deficient and control cells (Fig. 2e). However, and in line with previous reports, PTPN2-deficient cells expressed less $I L-8$ mRNA in response to TNF- $\alpha$ when compared to control cells $(p<0.001)$. Since IFN- $\gamma$-treatment had little or no effect in the so far analyzed genes, we confirmed response to IFN- $\gamma$ via analysis of mRNA levels of intercellular adhesion molecule-1. Intercellular adhesion molecule-1 mRNA levels were highly increased upon IFN- $\gamma$ stimulation in PTPN2-competent and even more in PTPN2-deficient IECs ( $p<0.001$, Fig. 2f). This was also true for TNF- $\alpha$ stimulation $(p<0.001)$.

\section{PTPN23 mRNA Expression is Increased in Colon} Lysates from DSS-Treated PTPN2xVilCre Mice

To further address the hypothesis that loss of PTPN2 might be compensated by altered expression of other ty-
18
Ulugöl et al. 
rosine phosphatases, we next measured mRNA expression of Ptpn2, Ptpn1, Ptpn9, Ptpn11, Ptpn22, and Ptpn23 in colon tissue samples of wildtype (wt) mice and mice lacking PTPN2 specifically in IECs (PTPN2xVilCre mice). The mice had either been left untreated or treated with 2\% DSS in the drinking water for 7 days to induce an acute colitis [17]. As already described in Kasper et al. [17], this treatment resulted in similar intestinal inflammation in wt and PTPN2-VilCre mice.

As depicted in Figure 3, mRNA expression of Ptpn2 was significantly reduced in colon pieces from PTPN2xVilCre mice, and loss of PTPN2 also resulted in a marked reduction of Ptpn1 mRNA levels (Fig. 3a, b). On the other hand, we did not observe any significant changes in Ptpn9 and Ptpn11 mRNA expression in PTPN2xVilCre mice when compared to wt animals (Fig. 3c, d). Whereas DSS-treated wt animals showed slightly enhanced Ptpn 22 mRNA expression when compared to their control littermates without DSS, its expression was significantly decreased in PTPN2xVilCre mice that were treated with DSS ( $p<0.01$, Fig. 3e).

Of note, while Ptpn 23 mRNA was not altered in PTPN2xVilCre mice without induction of colitis, there was a clearly up-regulation of Ptpn 23 upon DSS treatment in PTPN2-xVilCre mice ( $p<0.01$, Fig. 3f). Up-regulation of PTPN23 in PTPN2-VilCre mice was further confirmed in IHC staining of distal colon sections (Fig. 3g).

We have previously shown that loss of PTPN2 promotes activation of inflammasomes [18], and subsequent secretion of IL- $1 \beta$ and IL- 18 . Therefore, we also addressed whether loss of PTPN2 in epithelial cells affects expression of $I l 1 b$ and $I l 18$ mRNA. While Il $1 b$ mRNA expression level was not altered in all 4 groups, Il18 mRNA expression showed a trend toward a reduction in DSS-treated wt and PTPN2xVilCre mice compared to their corresponding controls (Fig. 3h, i), possibly due to a negative feedback regulation. This hypothesis was confirmed by addressing IL-18 levels in serum by ELISA, where we found increased IL-18 protein levels in DSS-treated WT mice when compared to water controls. Of note, this increase was further enhanced in DSS-treated PTPN2-VilCre mice (Fig. 3j). This is in line with our previous finding that the regulation of IL- $1 \beta$ and IL- 18 via PTPN2 is mediated via changes in protein activation, and not via changes in mRNA levels [18].

mRNA Expression Level in Colon Lysates from AOMDSS Treated PTPN2xVilCre Mice

In a next step, we aimed to address whether loss of PTPN2 also affects expression of other phosphatases in a model of colitis-associated tumor formation. For this aim, wt mice and PTPN2xVilCre littermates were subjected to a commonly used mouse model for colorectal carcinoma, namely, the AOM/DSS induced colon tumor model [19]. This treatment resulted in colon inflammation and tumor development as previously described in Spalinger et al. [18]. In this experiment, colon tissue pieces from nontumor tissue and from tumor tissue have been collected for mRNA analysis and we here investigated the expression levels of Ptpn2, Ptpn1, Ptpn9, Ptpn11, Ptpn22, Ptpn23, Illb, and Ill8 mRNA.

Ptpn2 mRNA expression was decreased in PTPN2xVilCre mice, but not affected by DSS/AOM treatment in tumor or nontumor tissue (Fig. 4a). As shown in Figure 4b, Ptpn 1 mRNA expression tended to be higher in nontumor tissue from mice treated with AOM/DSS than in control littermates, although this effect was not statistically significant. Regardless of the genotype, there was significantly more Ptpn1 expressed in tumor tissue than in colon tissue from healthy wt mice ( $p<0.01$, Fig. $4 \mathrm{~b})-$ this was in contrast to our findings obtained using acute DSS colitis model, where Ptpn1 expression was markedly decreased upon deletion of PTPN2. In contrast to our cell culture experiment, but in line with results from the DSS acute colitis, Ptpn9 expression was not affected by AOM/ DSS treatment and did not show an altered mRNA expression profile. Likewise, Ptpn 11 mRNA levels were not affected by treatment or genotype (Fig. 4c, d).

Ptpn 22 mRNA expression was increased in nontumor colon tissue from AOM/DSS-treated PTPN2xVilCre mice compared to healthy PTPN2xVilCre $(p<0.05)$ and wt control mice ( $p<0.01$, Fig. $4 \mathrm{e})$. Upon AOM/DSS treatment, Ptpn 22 expression was significantly decreased in tumor tissue from wt mice, when compared to nontumor tissue from the same mice ( $p<0.05$, Fig. 4e). Although there seems also to be a decrease of Ptpn 22 expression in tumor tissue from PTPN2xVilCre mice, this reduction was far less pronounced, and tumor tissue from PTPN2xVilCre mice tended to express more Ptpn22 than wt tumor tissue (Fig. 4e).

In the colon tumor mouse model, we observed that Ptpn 23 mRNA expression was clearly increased in colonic nontumor tissue derived from PTPN2xVilinCre mice after AOM/DSS treatment compared to wt control mice $(p<0.01$, Fig. 4f) and wt mice treated with AOM/DSS ( $p<$ $0.05)$. Interestingly, Ptpn 23 mRNA expression was not altered in tumor tissue of neither PTPN2 wt nor PTPN2xVilCre mice when compared to the respective untreated control mice. mRNA expression data were then confirmed by IHC, where we found increased HD-PTP ex- 


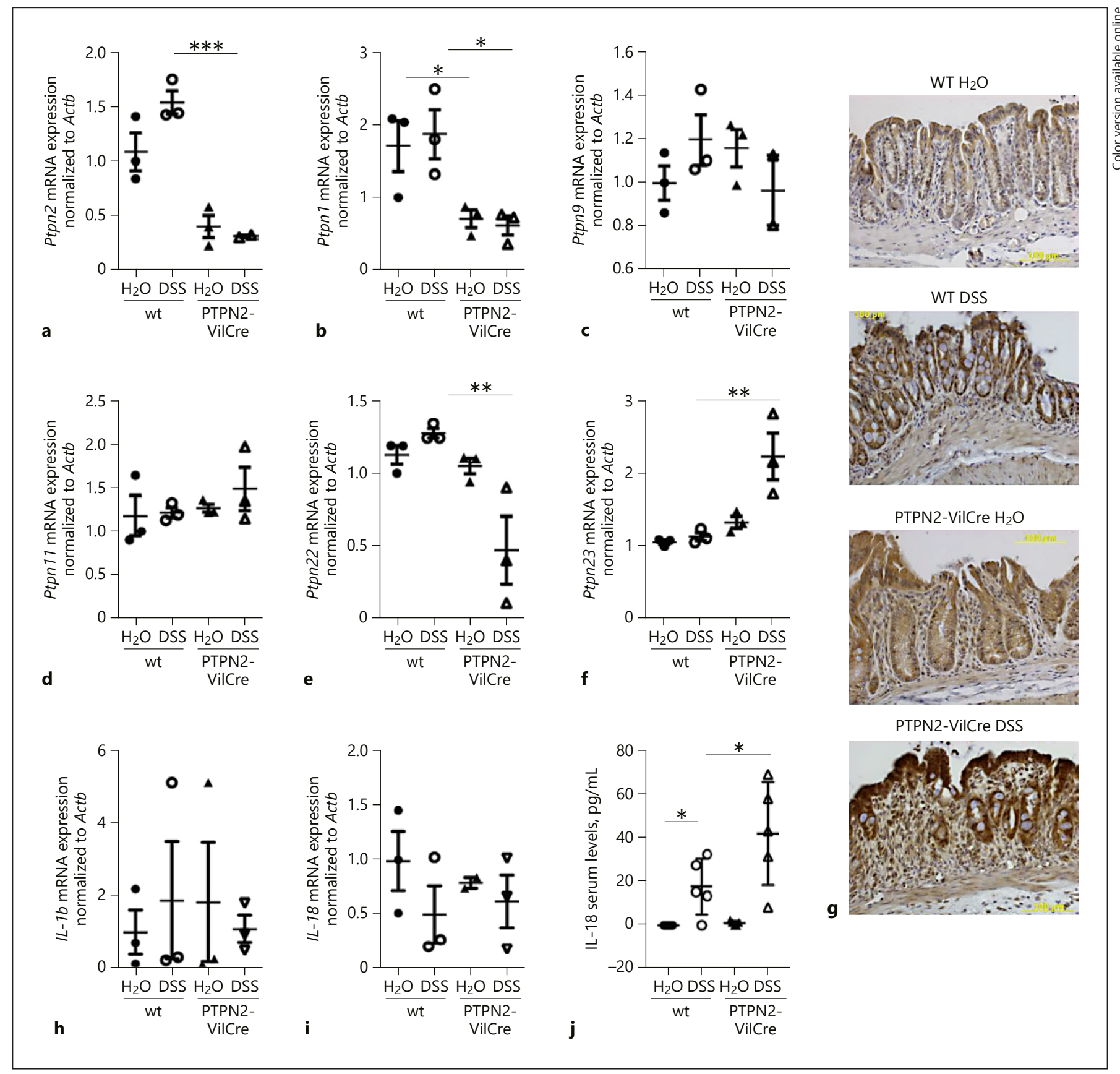

Fig. 3. Colon lysates from DSS-treated PTPN2xVilCre mice show increased expression of PTPN23. C57/Bl6 mice with a floxed Ptpn2 gene (wt) and PTPN2fl/fl mice expressing Cre-recombinase under the Villin promoter that lack Ptpn 2 specifically in IECs (PTPN2xVilCre mice) were treated with water or $2 \%$ DSS. Colon lysates of PTPN2xVilCre mice were analyzed for mRNA expression of (a) Ptpn2, (b) Ptpn1, (c) Ptpn9, (d) Ptpn11, (e) Ptpn22, and (f) Ptpn23 by RT-PCR. Data were normalized to Actb. $\mathbf{g}$ Paraffin-embedded tissue from the terminal colon was stained for HD-PTP (brown), the protein product of PTPN23. mRNA expression of (h) IL-1b and (i) IL18 were analyzed by RT-qPCR, and (j) serum IL-18 levels detected by ELISA. Each symbol stands for one individual mouse and horizontal bars indicate the mean. Asterisks denote significant differences * $p<0.05,{ }^{* *} p<0.01,{ }^{* * *} p<0.001$ Newman Keul's test; wt, wildtype; DSS, dextran sulfate sodium; Actb, actin beta (housekeeping gene, mouse); PTPN, protein tyrosine phosphatase nonreceptor type. 

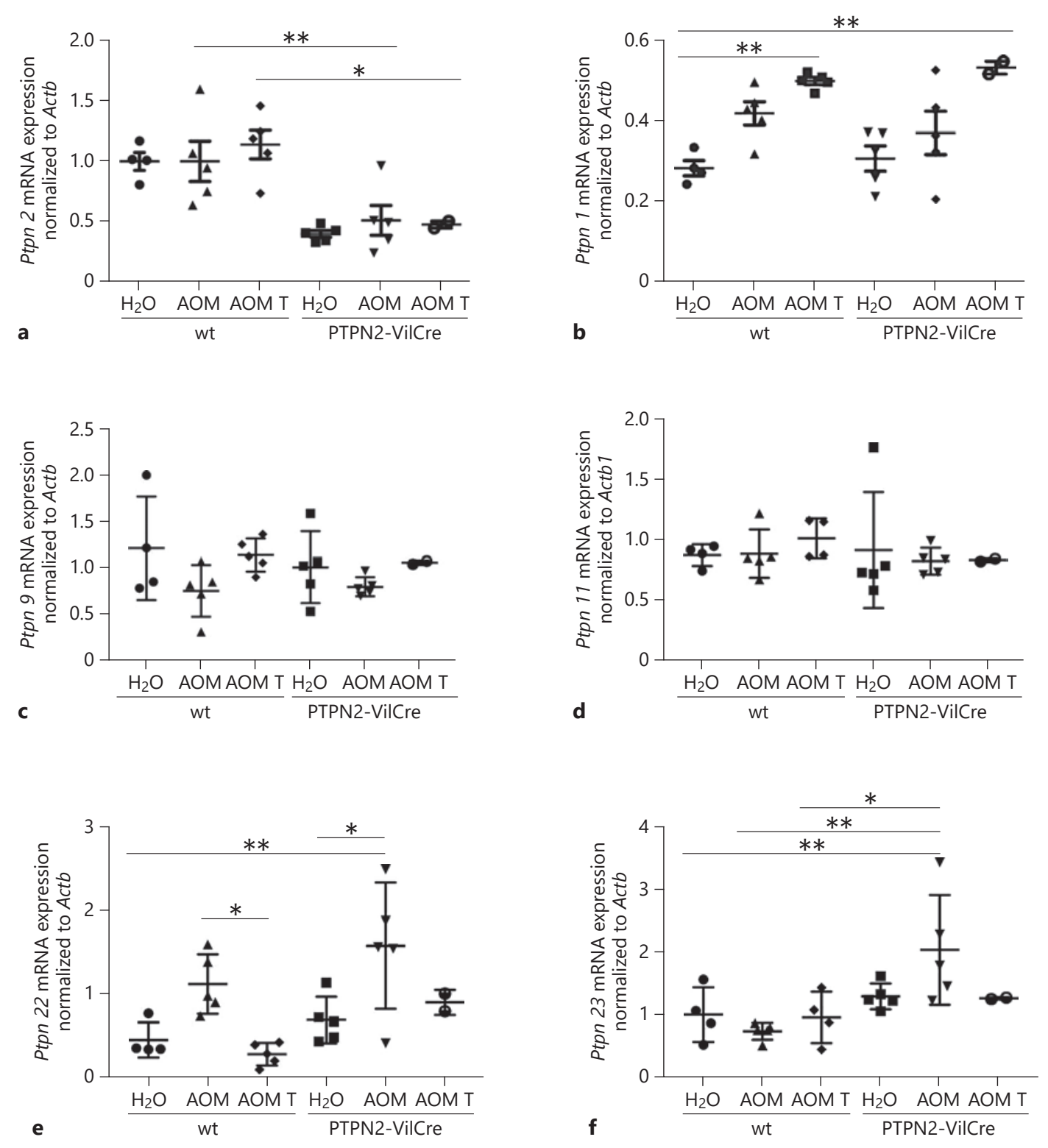

Fig. 4. PTPN23 expression is enhanced in PTPN2xVilCre mice after AOM/DSS treatment but not increased in tumor tissue. To induce colitis-associated tumors, PTPN2xVilCre mice were treated for 3 consecutive cycles consisting of 7 days with $1.5 \%$ DSS followed by 10 days recovery phase, each. At the first day of each cycle, $10 \mathrm{mg} / \mathrm{kg}$ body weight AOM was injected. Colon lysates and tumor pieces were analyzed for mRNA expression of (a) PTPN2,

(b) PTPN1, (c) PTPN9, (d) PTPN11, (e) PTPN22, and (f) PTPN23 by RT-PCR. Data were normalized to Actb. * Significant differences ${ }^{*} p<0.05,{ }^{* *} p<0.01,{ }^{* * *} p<0.001$ Newman Keul's test; wt, wildtype; ko, knockout; AOM, azoxymethane; AOM T, azoxymethane-treated group, tumor tissue; PTPN, protein tyrosine phosphatase nonreceptor type.

pression in PTPN2-VilCre mice in general, and a slightly more pronounced increase in tumor tissue from PTPN2VilCre mice (Fig. 5).

When analyzing the expression of $I l 1 b$ and $I l 18$, we observed that in general, $I l 1 b$ mRNA was upregulated

upon AOM/DSS treatment (Fig. 6a, b). The strongest increase in expression was found in tumor tissue of wt mice, followed by tumor tissue of PTPN2-VilCre mice. In contrast to $I l 1 b, I l 18$ mRNA expression was reduced in wt mice treated with AOM/DSS $(p<0.01)$ and in tumor tis- 


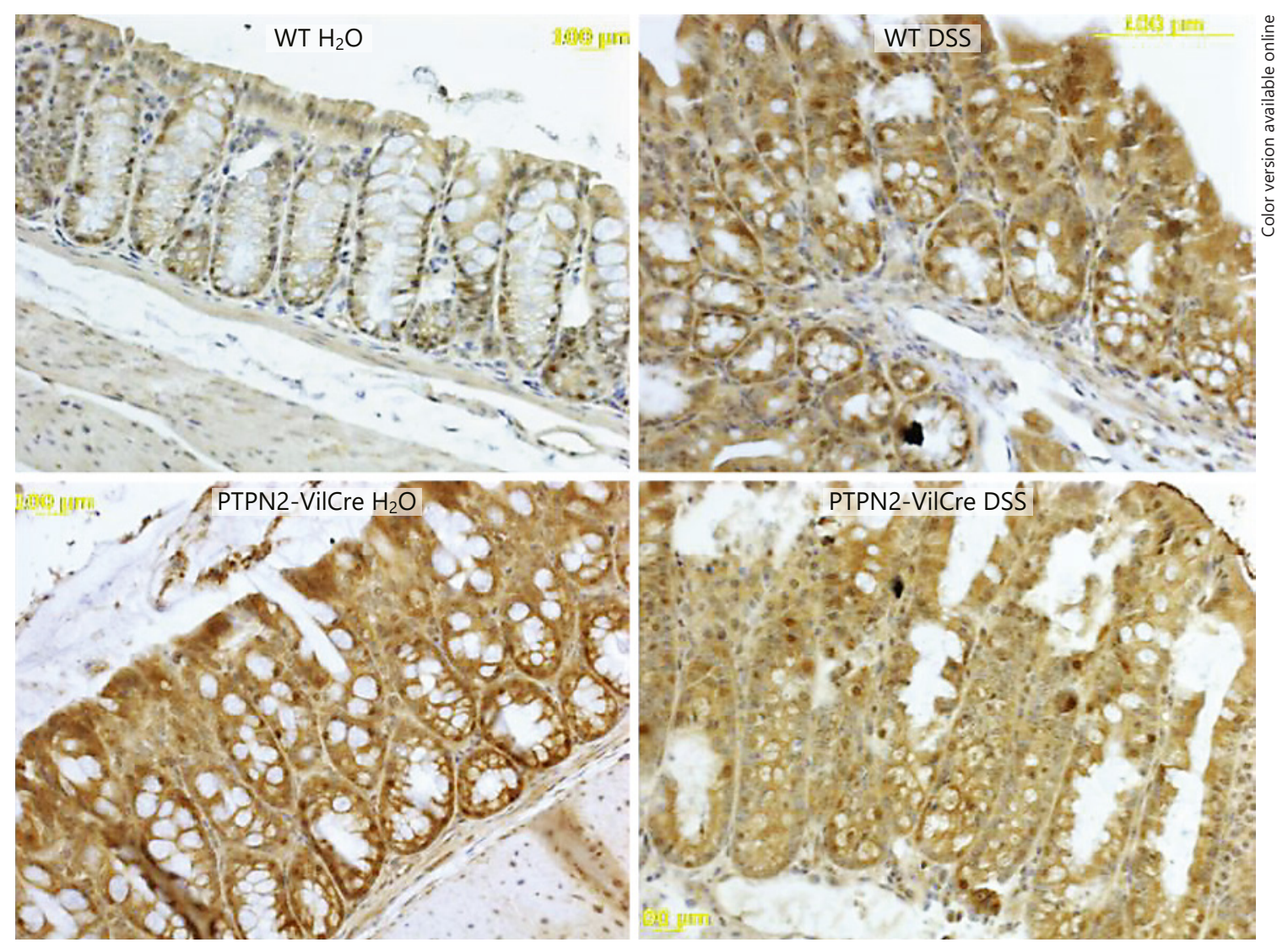

Fig. 5. PTPN23 protein expression is enhanced in PTPN2xVilCre mice after AOM/DSS treatment. To induce colitis-associated tumors, PTPN2xVilCre mice were treated for 3 consecutive cycles consisting of 7 days with 1.5\% DSS followed by 10 days recovery phase, each. At the first day of each cycle, $10 \mathrm{mg} / \mathrm{kg}$ body weight
AOM was injected. Paraffin-embedded tissue from the terminal colon was stained for HD-PTP (brown), the protein product of PTPN23. wt, wildtype; DSS, dextran sulfate sodium; PTPN, protein tyrosine phosphatase nonreceptor type. sue from wt $(p<0.01)$ and PTPN2xVilCre mice $(p<0.05)$. Surprisingly, Il18 mRNA expression was highest in wt control mice when compared to all other groups, which again might result from a negative feedback regulation. This hypothesis was confirmed using ELISA for IL-18, where we found a clear trend toward increased serum levels in AOM/DSS-treated PTPN2-VilCre mice (Fig. 6c).

\section{Discussion}

In this study, we investigated how loss of PTPN2 in IECs modulates the expression of related tyrosine phosphatases in HT-29 cells, colon lysates, and tumor tissue of PTPN2xVilCre mice. Our results showed that in vivo, loss of PTPN2 in IECs mainly affected the expression of Ptpn 23 mRNA, while Ptpn 9 and Ptpn 11 were not changed in a consistent manner among the different experimental settings. This was supported by our HT29 cell line studies, where we identified as a most striking finding a regulatory loop between PTPN2 and PTPN23 in IECs (Fig. 7).

In the in vivo setting, we found that among the studied tyrosine phosphatases, only PTPN23 was more expressed in tissue of PTPN2xVilCre mice compared to their wt littermates. This result is consistent with our finding in the in vitro experiments, where PTPN23 mRNA expression was also increased in all 3 groups of HT29-PTPN2-knockdown cells compared to the respective control groups. It has been shown that PTPN23 is essential during embryonic development, and its expression in adult mice is limited to the epithelium of multiple organs including the epithelium of the stomach, small intestine, and colon [20]. PTPN23 encodes for the protein HD-PTP, which controls cell migration and endocytosis [21]. The role of PTPN23 in IECs in the intestine has not been studied yet. However, our recent unpublished findings indicate that 

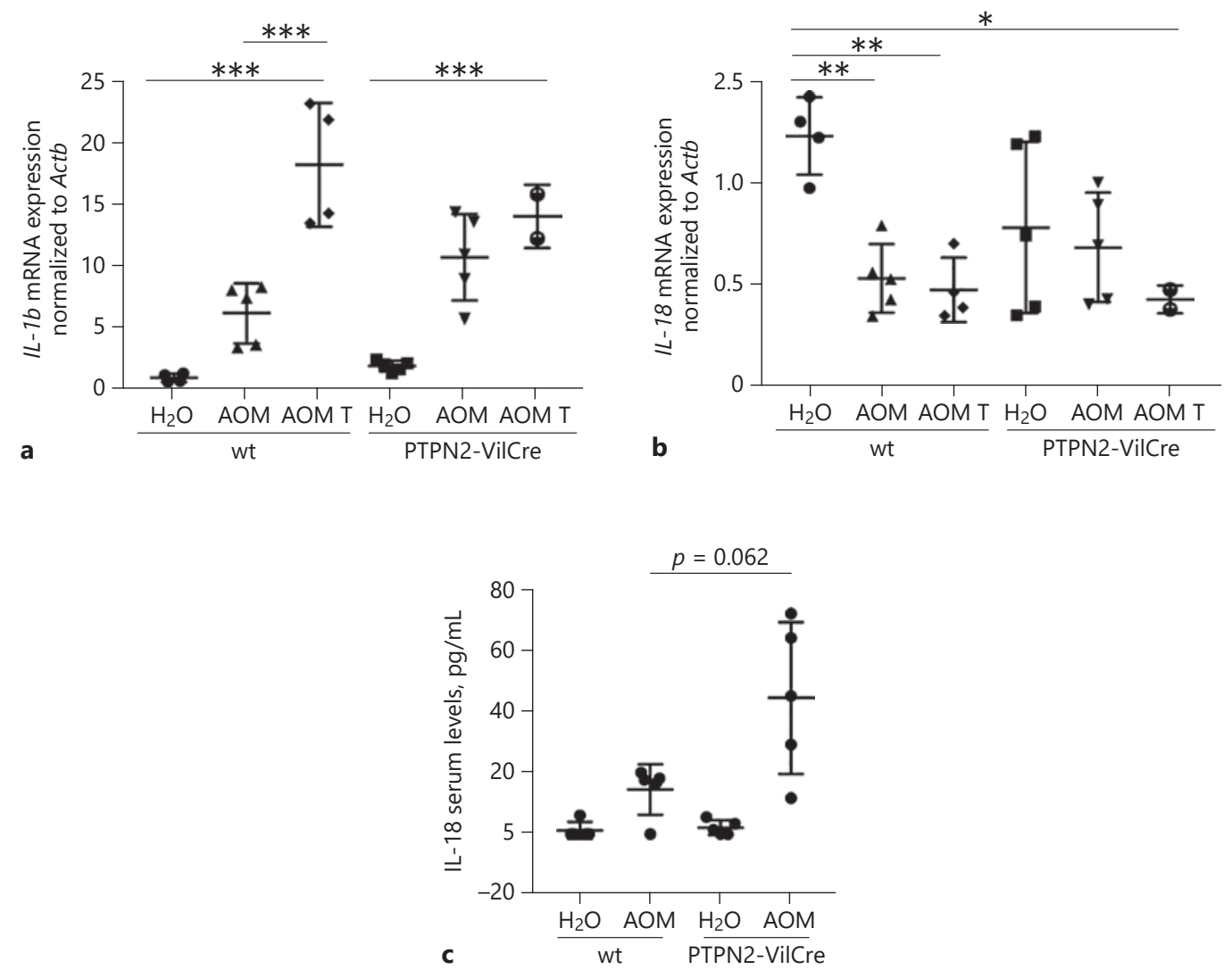

Fig. 6. Altered expression of inflammatory cytokines in PTPN2xVilCre mice after AOM/DSS treatment. Mice were treated as in Figure 4 and colon lysates and tumor pieces analyzed for (a) IL-1b and (b) IL-18 mRNA expression. Data were normalized to Actb. Serum from the same mice was analyzed for IL-18 protein levels

Fig. 7. Proposed mechanism how PTPN23 might counteracts the effect of PTPN2 deficiency. Deletion of PTPN2 promotes pro-inflammatory and pro-oncogenic signaling cascades, such as JAK/ STAT signaling, EGFR signaling, and IL-18 secretion. At the same time, it promotes the expression of PTPN23. PTPN23 in turn inhibits EGFR signaling, and IL-18 induced effects, thus counteracting the effects of PTPN2 deficiency. Blue arrows: enhanced expression, red arrows with blunt ends: suppression. Dashed lines: proposed effects, solid lines: effects supported by literature. PTPN, protein tyrosine phosphatase nonreceptor type.

PTPN23/HD-PTP might control proliferation and migration of IECs.

Interestingly, PTPN23 expression was only increased in nontumor tissue from PTPN2xVilCre mice treated with AOM/DSS but not in the tumor tissue itself. This (c). Significant differences ${ }^{*} p<0.05,{ }^{* *} p<0.01,{ }^{* * *} p<0.001 \mathrm{New}$ man Keul's test; wt, wildtype; ko, knockout; AOM, azoxymethane; AOM T, azoxymethane-treated group, tumor tissue; PTPN, protein tyrosine phosphatase nonreceptor type; Actb, actin beta.

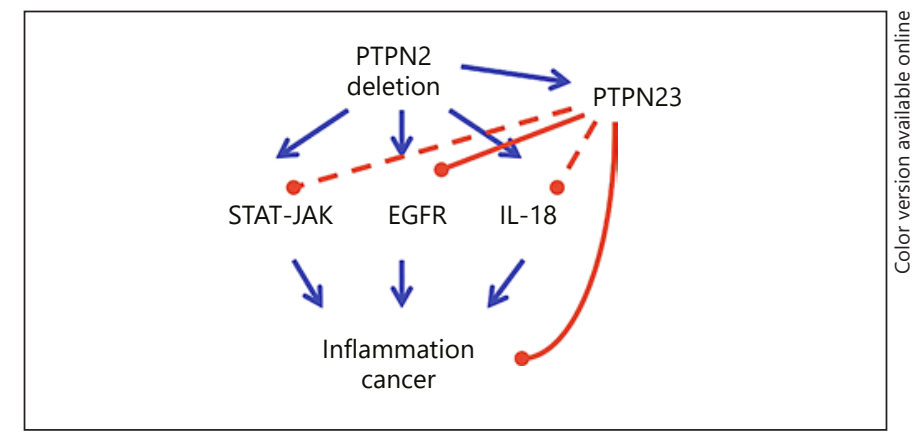

indicates that in the course of tumor formation and progression, PTPN23 upregulation seems to be lost and therefore also its compensatory function. This is in line with several studies that show that loss of PTPN23 promotes the development of breast cancer and that hemizy- 
gous loss of PTPN23 facilitates spontaneous tumorgenesis $[22,23]$.

A very interesting observation is that in PTPN2-deficient cells, the mRNA expression pattern of IL-18 follows the expression pattern of PTPN23. This is in contrast to $I l 1 b$ mRNA levels. Those findings suggest that in absence of PTPN2, PTPN23 might play a critical role for the regulation of IL-18 expression, and it might be interesting to investigate whether PTPN23 might be involved in the regulation of IL-18 protein expression and activation as well.

In addition to PTPN23 upregulation, PTPN22 mRNA expression was reduced in PTPN2xVilCre mice upon DSS treatment. We have previously shown that PTPN22 is reduced in the intestine of IBD patients and that it plays a protective role during intestinal inflammation [24]. Further, PTPN22-deficient mice are more affected by DSS-induced colitis [25-27]. One possible explanation for reduced PTPN22 expression might be the fact that TNF- $\alpha$ and IL- $1 \beta$ suppress PTPN22 expression [28]. The findings that PTPN22 was clearly reduced in the tumor tissue compared to the nontumor tissue in wild-type and PTPN2xVilCre mice suggests that PTPN22 might also exert somehow a tumor suppressor function in the colon regardless of PTPN2.

Of note, we found reduced PTPN1 mRNA expression in PTPN2xVilCre mice in the acute DSS experiment, regardless whether the mice were treated with DSS or not, while this was not the case in the AOM-DSS experiment. This seems contradictory at first, but might be explained by the fact that the mice are several weeks older at the end of the AOM-DSS tumor induction. We showed that in vitro, PTPN1 expression is clearly regulated upon presence of inflammatory cytokines. Lowgrade inflammatory processes might therefore account for a lack of genotype-specific differences in older mice. PTPN1 is closely related to PTPN2, sharing approximately $70 \%$ of their catalytic domain sequence [29]. Since PTPN1 is a negative regulator of JAK/STAT-mediated signaling regulating myeloid-derived suppressor cells, PTPN1 null mice are more resistant to DSS-induced colitis due to expansion of protecting myeloidderived suppressor cells that modulate cytokine secretion [30]. Therefore, the marked reduction of PTPN1 in our acute experiments might also contribute to the fact that PTPN2xVilCre mice do not suffer from pronounced acute colitis.

We observed that PTPN9 mRNA was highest in untreated PTPN2-deficient HT-29 cells. Its expression decreased strongly upon stimulation with the proinflamma- tory cytokines IFN- $\gamma$ and TNF- $\alpha$. However, the expression of PTPN9 mRNA was not changed in PTPN2xVilCre mice during induction of colitis, and it is well possible that other inflammatory cytokines, such as IL- 6 or IL-1 might counteract the effects of TNF- $\alpha /$ IFN- $\gamma$ in the in vivo situation. Hence, further studies would be necessary to fully understand the regulation of PTPN9 in the (inflamed) intestine.

Interestingly, we found increased expression of PTPN11 in PTPN2-deficient HT29 cells upon TNF- $\alpha$ stimulation but not upon IFN- $\gamma$ treatment. This might be explained by the regulatory function of PTPN2 on IFN- $\gamma$-induced signaling cascades [31]. Several studies proposed that PTPN11 is a protective factor against gut inflammation $[28,32]$, and its upregulation in IECs might represent a compensatory effect for the loss of PTPN2. Nevertheless, we did not observe significant differences in PTPN11 mRNA expression in colon lysates from PTPN2xVilCre mice upon colitis induction, leading to the conclusion that this phosphatase might play a subordinate role to compensate for the loss of PTPN2 in vivo. Further, in the in vivo situation, only IEC lack PTPN2, while all other cells express normal PTPN2 levels. In our study, we measured RNA in whole colon tissue, which includes IEC, as well as myeloid and lymphoid immune cells. Although our results show that many of the in vitro effects are mirrored in the wholetissue studies, the effect on PTPN11 expression observed in the samples from the in vivo experiment might reflect the effects resulting from non-IEC cells. Given the complex interaction between phosphatases and various intracellular signaling molecules, network analyses might be an interesting further direction to more completely understand how loss of PTPN2 in IEC affects cellular signaling pathways as a whole, as well as possible interaction between different cell types present in the intestine.

It should be considered that in our cell culture experiment, knockdown of PTPN2 was not complete; hence, the effects in the in vitro experiments might be less pronounced than from a complete knockout and might more closely reflect the situation observed in patients where presence of the variants results in reduced PTPN2 function but not its complete loss.

Taken together, our results demonstrate that depletion of PTPN2 in IECs affects the expression of other tyrosine phosphatases, which might contribute to the fact that mice lacking PTPN2 specifically in IECs do not develop pronounced colitis. Most strikingly, there was a marked and consistent up-regulation of PTPN23 in PTPN2-defi-
Ulugöl et al. 
cient cells and in colon tissue from PTPN2xVilCre mice, indicating that in vivo, this phosphatase might be the most relevant to compensate for the lack of PTPN2.

\section{Disclosures Statement}

The authors declare no conflicts of interest.

\section{Statement of Ethics}

All animal experiments were performed according to local animal welfare legislation and were approved by the Veterinary Office of the Canton Zurich (ZH-255/2014).

\section{Funding Source}

This research has been supported by grants to MiS from the Promedica foundation, the Stiftung Experimentelle Biomedizin, and the Swiss National Science Foundation (Grant No. 314730_166381).

\section{Author Contributions}

S.U., L.H., C.G., S.L., R.M., M.R.S.: data acquisition and interpretation, statistical analyses; M.S., M.R.S.: study design, supervision of experiments; S.U.: writing of first manuscript draft; all author edited, corrected, and approved the manuscript.

\section{References}

1 Beaugerie L, Itzkowitz SH. Cancers Complicating Inflammatory Bowel Disease. N Engl J Med. 2015 Jul;373(2):195.

2 Axelrad JE, Lichtiger S, Yajnik V. Inflammatory bowel disease and cancer: the role of inflammation, immunosuppression, and cancer treatment. World J Gastroenterol. 2016 May; 22(20):4794-801.

3 Zhang YZ, Li YY. Inflammatory bowel disease: pathogenesis. World J Gastroenterol. 2014 Jan;20(1):91-9.

4 Peters LA, Perrigoue J, Mortha A, Iuga A, Song WM, Neiman EM, et al. A functional genomics predictive network model identifies regulators of inflammatory bowel disease. Nat Genet. 2017 Oct;49(10):1437-49.

5 Franke A, McGovern DP, Barrett JC, Wang K, Radford-Smith GL, Ahmad T, et al. Genome-wide meta-analysis increases to 71 the number of confirmed Crohn's disease susceptibility loci. Nat Genet. 2010 Dec;42(12): 1118-25.

6 de Lange KM, Moutsianas L, Lee JC, Lamb CA, Luo Y, Kennedy NA, et al. Genome-wide association study implicates immune activation of multiple integrin genes in inflammatory bowel disease. Nat Genet. 2017 Feb; 49(2):256-61.

7 Lees CW, Barrett JC, Parkes M, Satsangi J. New IBD genetics: common pathways with other diseases. Gut. 2011 Dec;60(12):173953.

8 Lee H, Yi JS, Lawan A, Min K, Bennett AM. Mining the function of protein tyrosine phosphatases in health and disease. Semin Cell Dev Biol. 2015 Jan;37:66-72.

9 Bussières-Marmen S, Hutchins AP, Schirbel A, Rebert N, Tiganis T, Fiocchi C, et al. Characterization of PTPN2 and its use as a biomarker. Methods. 2014 Jan;65(2):239-46.

10 You-Ten KE, Muise ES, Itié A, Michaliszyn E, Wagner J, Jothy S, et al. Impaired bone marrow microenvironment and immune function in $\mathrm{T}$ cell protein tyrosine phosphatase- deficient mice. J Exp Med. 1997 Aug;186(5): 683-93.

11 Khor B, Gardet A, Xavier RJ. Genetics and pathogenesis of inflammatory bowel disease. Nature. 2011 Jun;474(7351):307-17.

12 Michielan A, D'Incà R. Intestinal Permeability in Inflammatory Bowel Disease: Pathogenesis, Clinical Evaluation, and Therapy of Leaky Gut. Mediators Inflamm. 2015;2015: 628157.

13 Coskun M. Intestinal epithelium in inflammatory bowel disease. Front Med (Lausanne). 2014 Aug; 1:24.

14 Beaurepaire C, Smyth D, McKay DM. Interferon-gamma regulation of intestinal epithelial permeability. J Interferon Cytokine Res. 2009 Mar;29(3):133-44.

15 Scharl M, Paul G, Weber A, Jung BC, Docherty MJ, Hausmann M, Rogler G, Barrett $\mathrm{KE}, \mathrm{McCole}$ DF. Protection of epithelial barrier function by the Crohn's disease associated gene protein tyrosine phosphatase n2. Gastroenterology. 2009 Dec;137(6):2030-2040.e5.

16 Scharl M, McCole DF, Weber A, Vavricka SR, Frei P, Kellermeier S, et al. Protein tyrosine phosphatase $\mathrm{N} 2$ regulates $\mathrm{TNF} \alpha$-induced signalling and cytokine secretion in human intestinal epithelial cells. Gut. 2011 Feb;60(2): 189-97.

17 Kasper SH, Spalinger MR, Leonardi I, Gerstgrasser A, Raselli T, Gottier C, et al. Deficiency of Protein Tyrosine Phosphatase Non-Receptor Type 2 in Intestinal Epithelial Cells Has No Appreciable Impact on Dextran Sulphate Sodium Colitis Severity But Promotes Wound Healing. Digestion. 2016;93(4):249-59.

18 Spalinger MR, Manzini R, Hering L, Riggs JB, Gottier C, Lang S, et al. PTPN2 Regulates Inflammasome Activation and Controls Onset of Intestinal Inflammation and Colon Cancer. Cell Rep. 2018 Feb;22(7):1835-48.

19 Parang B, Barrett CW, Williams CS. AOM/ DSS Model of Colitis-Associated Cancer. Methods Mol Biol. 2016;1422:297-307.
20 Gingras MC, Kharitidi D, Chénard V, Uetani N, Bouchard M, Tremblay ML, et al. Expression analysis and essential role of the putative tyrosine phosphatase His-domaincontaining protein tyrosine phosphatase (HD-PTP). Int J Dev Biol. 2009;53(7):106974.

21 Gahloth D, Heaven G, Jowitt TA, Mould AP, Bella J, Baldock C, et al. The open architecture of HD-PTP phosphatase provides new insights into the mechanism of regulation of ESCRT function. Sci Rep. 2017 Aug;7(1): 9151.

22 Zhang S, Fan G, Hao Y, Hammell M, Wilkinson JE, Tonks NK. Suppression of protein tyrosine phosphatase N23 predisposes to breast tumorigenesis via activation of FYN kinase. Genes Dev. 2017 Oct;31(19):1939-57.

23 Manteghi S, Gingras MC, Kharitidi D, Galarneau L, Marques M, Yan M, et al. Haploinsufficiency of the ESCRT Component HD-PTP Predisposes to Cancer. Cell Rep. 2016 May; 15(9):1893-900.

24 Spalinger MR, Lang S, Vavricka SR, Fried M, Rogler G, Scharl M. Protein tyrosine phosphatase non-receptor type 22 modulates NOD2-induced cytokine release and autophagy. PLoS One. 2013 Aug;8(8):e72384.

25 Chang HH, Miaw SC, Tseng W, Sun YW, Liu CC, Tsao HW, et al. PTPN22 modulates macrophage polarization and susceptibility to dextran sulfate sodium-induced colitis. J Immunol. 2013 Sep;191(5):2134-43.

26 Spalinger MR, Kasper S, Gottier C, Lang S, Atrott K, Vavricka SR, et al. NLRP3 tyrosine phosphorylation is controlled by protein tyrosine phosphatase PTPN22. J Clin Invest. 2016 Nov;126(11):4388

27 Wang Y, Shaked I, Stanford SM, Zhou W, Curtsinger JM, Mikulski Z, et al. The autoimmunity-associated gene PTPN22 potentiates toll-like receptor-driven, type 1 interferondependent immunity. Immunity. 2013 Jul; 39(1):111-22. 
28 Spalinger MR, Scharl M. The role for protein tyrosine phosphatase non-receptor type 22 in regulating intestinal homeostasis. United European Gastroenterol J. 2016 Jun;4(3):325-32.

29 Asante-Appiah E, Ball K, Bateman K, Skorey K, Friesen R, Desponts C, et al. The YRD motif is a major determinant of substrate and inhibitor specificity in T-cell protein-tyrosine phosphatase. J Biol Chem. 2001 Jul;276(28): 26036-43.
30 Zhang J, Wang B, Zhang W, Wei Y, Bian Z, Zhang CY, et al. Protein tyrosine phosphatase $1 \mathrm{~B}$ deficiency ameliorates murine experimental colitis via the expansion of myeloid-derived suppressor cells. PLoS One. 2013 Aug; 8(8):e70828.
31 McCole DF. Regulation of epithelial barrier function by the inflammatory bowel disease candidate gene, PTPN2. Ann N Y Acad Sci. 2012 Jun;1257(1):108-14.

32 Coulombe G, Rivard N. New and Unexpected Biological Functions for the Src-Homology 2 Domain-Containing Phosphatase SHP-2 in the Gastrointestinal Tract. Cell Mol Gastroenterol Hepatol. 2015 Nov;2(1):11-21. 